Supporting information for:

\title{
Titanium and Zirconium Complexes of Robust Salophan Ligands. Coordination Chemistry and Olefin Polymerization Catalysis
}

\author{
Shimrit Gendler, Ayellet L. Zelikoff, Jacob Kopilov, Israel Goldberg and Moshe Kol* \\ School of Chemistry, Raymond and Beverly Sackler Faculty of Exact Sciences, \\ Tel Aviv University, Ramat Aviv, Tel Aviv 69978, Israel.
}




\section{General}

All the experiments that required inert atmosphere were performed under an atmosphere of dry nitrogen in a nitrogen-filled glovebox. Ether and tetrahydrofuran were purified by reflux and distillation under dry argon atmosphere from $\mathrm{Na}$ /benzophenone. Pentane was washed with $\mathrm{HNO}_{3} / \mathrm{H}_{2} \mathrm{SO}_{4}$ prior to distillation from $\mathrm{Na} /$ benzophenone/tetraglyme. Toluene was refluxed over $\mathrm{Na}$ and distilled. Ethyl chloroformate, diamino benzene, 4,5-dichloro-1,2-diaminobenzene, 3,5-dichlorosalicylaldehyde, Hydrogen bromide 30 wt \% solution in acetic acid, $\mathrm{LiAlH}_{4}$, Benzyl magnesium chloride, Titanium(IV) isopropoxide, Zirconium(IV) tert-butoxide and 1-hexene were purchased from Aldrich Inc. Tris(pentafluorophenyl)borane was obtained from Strem Chemicals. 1-hexene was passed through alumina prior to use. Tetrabenzylzirconium ${ }^{1}$ and 2-(bromomethyl)-4,6-bis(1,1-dimethylethyl)phenol ${ }^{2}$ were synthesized according to published procedures. NMR data were recorded on a Bruker AC-200 and a Bruker Avance AC-400 spectrometers and referenced to protio impurities in benzene- $\mathrm{d}_{6}$, chloroform$\mathrm{d}$, and DMSO- $\mathrm{d}_{6}\left(\delta 7.15,7.26\right.$ and 2.50, respectively), and to ${ }^{13} \mathrm{C}$ chemical shift of benzene, chloroform and DMSO ( $\delta$ 128.70, 77.16 and 39.52, respectively). Elemental analyses were performed in the microanalytical laboratory in the Hebrew University in Jerusalem. The metal complexes were analyzed within a few hours of being taken out of the freezer of the glove box. The molecular weights of poly(1hexene) were determined by gel permeation chromatography (GPC) using TSKgel GMHHR-M, TSKgel and G 4000 HHR columns set on a Jasco instrument equipped with a refractive index detector. Molecular weight determination was carried out relative to polystyrene standards using tetrahydrofuran (HPLC grade, distilled and filtered under vacuum prior to use) as the eluting solvent. The X-ray diffraction measurements were performed on a Nonius Kappa CCD diffractometer system, using MoKa $(\lambda=0.7107 \AA)$ radiation. The analyzed crystals were embedded within a drop of viscous oil (Paratone$\mathrm{N})$ and freeze-cooled to ca. $110 \mathrm{~K}$. The structures were solved by a combination of direct methods and Fourier techniques using the SIR-92 software, ${ }^{3}$ and were refined by full-matrix least squares with SHELXL-97. ${ }^{4}$ 
This compound was previously synthesized by a different approach. ${ }^{5}$

A solution of ethyl chloroformate $(5.9 \mathrm{~mL}, 70 \mathrm{mmol})$ in toluene $(20 \mathrm{~mL})$ was added dropwise to a cold solution of 1,2-diaminobenzene $(6.7 \mathrm{~g}, 70 \mathrm{mmol})$ in toluene $(20 \mathrm{~mL})$ and a white precipitate formed immediately. The reaction mixture was stirred at room temperature for $3 \mathrm{~h}$, the precipitate was removed by filtration, and the toluene was removed under vacuum to give a pink solid in a final yield of $59 \%$.

The spectral data of the product matches the data previously reported.

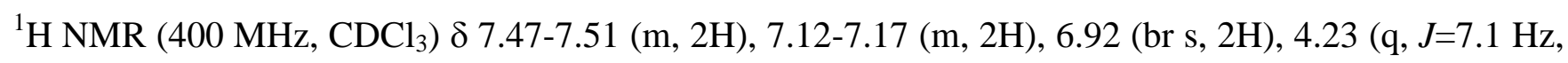
4H), $1.31(\mathrm{t}, J=7.1 \mathrm{~Hz}, 6 \mathrm{H}) .{ }^{13} \mathrm{C} \mathrm{NMR}\left(100.63 \mathrm{MHz}, \mathrm{CDCl}_{3}\right) \delta 154.9\left(2 \mathrm{C}, \mathrm{NHCO}_{2} \mathrm{Et}\right) 130.1(2 \mathrm{C}, C$, Arom), 125.6, 124.3 (4C, $\mathrm{CH}$, Arom), 61.7 (2C, $\left.\mathrm{CH}_{2} \mathrm{CH}_{3}\right), 14.5$ (2C, $\left.\mathrm{CH}_{2} \mathrm{CH}_{3}\right)$.

MS (EI): calc. for $\mathrm{C}_{12} \mathrm{H}_{16} \mathrm{~N}_{2} \mathrm{O}_{4}$ : 252, found: 252 .

\section{$\underline{N, N \text {-dimethyl-1,2-diaminobenzene }}$}

This compound was previously synthesized by a different approach. ${ }^{6}$

2-(Ethoxycarbonylamino-phenyl)-carbamic acid ethyl ester $(3.3 \mathrm{~g}, 13 \mathrm{mmol})$ was added in portions to a cold suspension of $\mathrm{LiAlH}_{4}(3.0 \mathrm{~g}, 78 \mathrm{mmol})$ in dry THF $(40 \mathrm{~mL})$ under argon atmosphere. The reaction mixture was stirred at room temperature for $1 \mathrm{~h}$, followed by $6 \mathrm{~h}$ of reflux. The mixture was cooled to 0 ${ }^{\circ} \mathrm{C}$ and then water $(3 \mathrm{~mL}), \mathrm{NaOH} 15 \%(3 \mathrm{~mL})$ and water $(9 \mathrm{~mL})$ were added. The mixture was stirred over night under argon atmosphere. The white precipitate was filtered off and extracted with warm THF, and the volatiles were removed under vacuum to give green oil that was acidified with $\mathrm{HCl} 10 \%$. The aqueous solution was washed three times with dichloromethane. The aqueous solution was basified with $\mathrm{NaOH} 10 \%$ and extracted three times with dichloromethane. The organic phase was dried over anhydrous $\mathrm{MgSO}_{4}$ and the solvent was removed under vacuum to give red oil in a final yield of $47 \%$. The spectral data matches the data previously reported.

${ }^{1} \mathrm{H}$ NMR $\left(200 \mathrm{MHz}, \mathrm{CDCl}_{3}\right) \delta 6.82-6.89(\mathrm{~m}, 2 \mathrm{H}), 6.68-6.73(\mathrm{~m}, 2 \mathrm{H}), 3.28(\mathrm{br} \mathrm{s}, 2 \mathrm{H}), 2.88(\mathrm{~s}, 6 \mathrm{H})$. 


\section{$\underline{\operatorname{Lig}^{1} \mathrm{H}_{2}}$}

A solution of 2-(bromomethyl)-4,6-bis(1,1-dimethylethyl)phenol (3.19 g, $11 \mathrm{mmol})$ in dry THF (5 mL) was added to a stirred solution of $N, N^{\prime}$-dimethyl-1,2-diaminobenzene $(0.73 \mathrm{~g}, 5.5 \mathrm{mmol})$ in dry THF (5 $\mathrm{mL})$ under argon atmosphere. A solution of triethylamine $(1.5 \mathrm{~mL})$ in dry THF $(2 \mathrm{~mL})$ was added dropwise and a precipitate had formed. The reaction mixture was stirred for $2 \mathrm{~h}$ in the dark under argon atmosphere. The purple precipitate was removed, and the solvent of the yellow filtrate was removed under vaccum forming a brown solid. The solid was redissolved in dichloromethane and washed three times with saturated $\mathrm{NaCl}$ solution. The organic phase was dried over anhydrous $\mathrm{MgSO}_{4}$ and the solvent was removed under vacuum to give the crude product as an off-white solid. The crude product was purified by flash chromatography on Silica gel $60 \mathrm{H}$ with a mixture of petroleum ether: dichloromethane in increasing polarity as eluent. The pure product was obtained as an off-white solid in a final yield of $40 \%$.

M.p. $153-154{ }^{\circ} \mathrm{C}$ (decomposed). ${ }^{1} \mathrm{H}$ NMR $\left(400 \mathrm{MHz}, \mathrm{C}_{6} \mathrm{D}_{6}\right) \delta 9.90$ (s, 2H), 7.43 (d, J=2.3 Hz, 2H), 6.99$7.02(\mathrm{~m}, 2 \mathrm{H}) 6.90-6.93(\mathrm{~m}, 2 \mathrm{H}) 6.88(\mathrm{~d}, J=2.3 \mathrm{~Hz}, 2 \mathrm{H}), 3.78(\mathrm{~s}, 4 \mathrm{H}), 2.46(\mathrm{~s}, 6 \mathrm{H}), 1.58(\mathrm{~s}, 18 \mathrm{H}), 1.31$ (s, 18H). ${ }^{13} \mathrm{C}$ NMR (100.63 MHz, $\left.\mathrm{C}_{6} \mathrm{D}_{6}\right) \delta 154.2,148.3$ 141.1, 136.6 (8C, C, Arom), 126.2, 125.0, 123.5, $123.1\left(8 \mathrm{C}, \mathrm{CH}\right.$, Arom), 122.4 (2C, $C$, Arom), $60.9\left(2 \mathrm{C}, \mathrm{CH}_{2}\right), 44.0\left(2 \mathrm{C}, \mathrm{NCH}_{3}\right), 35.4,34.3(4 \mathrm{C}$, $\left.C\left(\mathrm{CH}_{3}\right)_{3}\right), 31.9,30.1\left(12 \mathrm{C} \mathrm{C}\left(\mathrm{CH}_{3}\right)_{3}\right)$. MS (FAB): calc. for $\mathrm{C}_{38} \mathrm{H}_{56} \mathrm{~N}_{2} \mathrm{O}_{2}: 572$, found: 572. Anal. Calc. For $\mathrm{C}_{38} \mathrm{H}_{56} \mathrm{~N}_{2} \mathrm{O}_{2}$ : C, 79.67; H, 9.85; N, 4.89. Found: C, 79.42; H, 10.10; N, 4.64.

\section{$\underline{\text { 3,5-dichlorosalicylalcohol }}$}

$\mathrm{LiAlH}_{4}(1.00 \mathrm{~g}, 26 \mathrm{mmol})$ was added in portions to a cooled solution of 3,5-dichlorosalicylaldehyde $(3.30 \mathrm{~g}, 17 \mathrm{mmol})$ in methanol $(100 \mathrm{~mL})$. The reaction mixture was stirred at room temperature for $2 \mathrm{~h}$ and another portion of $\mathrm{LiAlH}_{4}(1.00 \mathrm{~g}, 26 \mathrm{mmol})$ was added. After $1 \mathrm{~h}$, most of the solvent was removed under vacuum and a mixture of water and ice was added. The mixture was cooled over night and the yellow crystals that formed were isolated by filtration in a final yield of $81 \%$. 
${ }^{1} \mathrm{H}$ NMR (400 MHz, DMSO-d 6 ) $\delta 7.16(\mathrm{~d}, J=2.5 \mathrm{~Hz}, 2 \mathrm{H}), 6.90(\mathrm{~d}, J=2.5 \mathrm{~Hz}, 2 \mathrm{H}), 4.34(\mathrm{~s}, 2 \mathrm{H}) .{ }^{13} \mathrm{C}$ NMR (100.63 MHz, DMSO-d 6 ) $\delta 151.9,129.7$ (2C, C, Arom), 126.1, 123.1, (2C, CH, Arom), 120.8, 119.2 (2C, $C$, Arom), $60.8\left(1 \mathrm{C}, \mathrm{CH}_{2}\right)$.

\section{$\underline{\text { 2-(bromomethyl)-4,6-di-chlorophenol }}$}

3,5-dichlorosalicylalcohol $(1.00 \mathrm{~g}, 5 \mathrm{mmol})$ was added to a solution of $30 \%$ hydrogen bromide in acetic acid $(10 \mathrm{~mL})$, and the reaction mixture was stirred at room temperature for $20 \mathrm{~h}$. The mixture was cooled to $0^{\circ} \mathrm{C}$, water and ice were added, and the white precipitate that formed was filtered to give the product in a final yield of $48 \%$.

${ }^{1} \mathrm{H}$ NMR $\left(200 \mathrm{MHz}, \mathrm{CDCl}_{3}\right) \delta 7.29(\mathrm{~d}, J=2.5 \mathrm{~Hz}, 1 \mathrm{H}), 7.23(\mathrm{~d}, J=2.5 \mathrm{~Hz}, 1 \mathrm{H}), 5.88$ (br s, 1H), 4.49 (s, 2H). ${ }^{13} \mathrm{C} \mathrm{NMR}\left(50.38 \mathrm{MHz}, \mathrm{CDCl}_{3}\right) \delta 148.4$ (1C, $C$, Arom) 129.7, 129.0 (2C, $C \mathrm{H}$, Arom), 126.8, 125.5, 121.0 (3C, $C$, Arom), $27.0\left(1 \mathrm{C}, \mathrm{CH}_{2} \mathrm{Br}\right)$.

\section{$\underline{\operatorname{Lig}^{2} \mathrm{H}_{2}}$}

A solution of 2-(bromomethyl)-4,6-dichlorophenol (1.82 g, $7.0 \mathrm{mmol})$ in dry THF (5 mL) was added to a stirred solution of $N, N^{\prime}$-dimethyl-1,2-diaminobenzene $(0.48 \mathrm{~g}, 3.5 \mathrm{mmol})$ in dry THF $(5 \mathrm{~mL})$ under argon atmosphere. A solution of triethylamine $(1.0 \mathrm{~mL})$ in dry THF $(2 \mathrm{~mL})$ was added dropwise and a precipitate had formed. The reaction mixture was stirred for $2 \mathrm{~h}$ in the dark under argon atmosphere. The purple precipitate was removed and the volatiles were removed under vacuum forming a sticky brown solid. The crude product was purified by flash chromatography on Silica gel $60 \mathrm{H}$ with a mixture of petroleum ether : chloroform in increasing polarity as eluent. The pure product was obtained as an offwhite solid in a final yield of $50 \%$.

M.p. $112{ }^{\circ} \mathrm{C}$ (decomposed). ${ }^{1} \mathrm{H}$ NMR $\left(400 \mathrm{MHz}, \mathrm{C}_{6} \mathrm{D}_{6}\right) \delta 9.16$ (br s, 2H), 7.11 (d, J=2.5 Hz, 2H), 6.82$6.85(\mathrm{~m}, 2 \mathrm{H}) 6.73-6.76(\mathrm{~m}, 2 \mathrm{H}) 6.71(\mathrm{~d}, J=2.4 \mathrm{~Hz}, 2 \mathrm{H}), 3.52(\mathrm{~s}, 4 \mathrm{H}), 2.20(\mathrm{~s}, 6 \mathrm{H}) .{ }^{13} \mathrm{C}$ NMR $(100.63$ $\left.\mathrm{MHz}, \mathrm{C}_{6} \mathrm{D}_{6}\right) \delta 151.7,147.7$ (4C, $C$, Arom), 129.3, 129.1, 126.5, (6C, CH, Arom), 125.3, 124.2 (4C, $C$, Arom), 122.8 (2C, $C H$, Arom), 122.6 (2C, $C$, Arom), $59.3\left(2 \mathrm{C}, C_{2}\right), 41.9\left(2 \mathrm{C}, \mathrm{NCH}_{3}\right) . \mathrm{MS}(\mathrm{FAB})$ : 
calc. for $\mathrm{C}_{22} \mathrm{H}_{20} \mathrm{Cl}_{4} \mathrm{~N}_{2} \mathrm{O}_{2}$ : 486, found: 486. Anal. Calc. For $\mathrm{C}_{22} \mathrm{H}_{20} \mathrm{Cl}_{4} \mathrm{~N}_{2} \mathrm{O}_{2}$ : C, 54.35; H, 4.15; N, 5.76.

Found: C, 54.34; H, 4.12; N, 5.43.

\section{4,5-dichloro-1,2-bis(ethoxycarbonylamino)benzene}

A solution of ethyl chloroformate $(1.1 \mathrm{~mL}, 11 \mathrm{mmol})$ in toluene $(10 \mathrm{~mL})$ was added dropwise to a cold solution of 4,5-dichloro-1,2-diaminobenzene $(2.0 \mathrm{~g}, 11 \mathrm{mmol})$ in toluene $(10 \mathrm{~mL})$ and a white precipitate formed immediately. The reaction mixture was stirred at room temperature for $5 \mathrm{~h}$, the precipitate was removed by filtration, and the toluene was removed under vacuum to give a brown solid in a final yield of $47 \%$.

${ }^{1} \mathrm{H}$ NMR $\left(200 \mathrm{MHz}, \mathrm{CDCl}_{3}\right) \delta 7.66(\mathrm{~s}, 2 \mathrm{H}), 6.92(\mathrm{br} s, 2 \mathrm{H}), 4.23(\mathrm{q}, J=7.1 \mathrm{~Hz}, 4 \mathrm{H}), 1.31(\mathrm{t}, J=7.1 \mathrm{~Hz}$, 6H). ${ }^{13} \mathrm{C} \mathrm{NMR}\left(50.38 \mathrm{MHz}, \mathrm{CDCl}_{3}\right) \delta 154.6\left(2 \mathrm{C}, \mathrm{NHCO}_{2} \mathrm{Et}\right) 129.3,128.8(4 \mathrm{C}, \mathrm{C}, \mathrm{Arom}), 125.3(2 \mathrm{C}$, $\mathrm{CH}$, Arom), $62.2\left(2 \mathrm{C}, \mathrm{CH}_{2} \mathrm{CH}_{3}\right), 14.5\left(2 \mathrm{C}, \mathrm{CH}_{2} \mathrm{CH}_{3}\right) . \mathrm{MS}$ (EI): calc. for $\mathrm{C}_{12} \mathrm{H}_{14} \mathrm{Cl}_{2} \mathrm{~N}_{2} \mathrm{O}_{4}$ : 321, found: 321.

\section{4,5-dichloro- $N, N^{\prime}$-dimethyl-1,2-diaminobenzene}

4,5-dichloro-1,2-bis(ethoxycarbonylamino)benzene $(1.20 \mathrm{~g}, 4 \mathrm{mmol})$ was added in portions to a cold suspension of $\mathrm{LiAlH}_{4}(0.85 \mathrm{~g}, 22 \mathrm{mmol})$ in dry THF $(20 \mathrm{~mL})$ under argon atmosphere. The reaction mixture was stirred at room temperature for $1 \mathrm{~h}$, followed by $6 \mathrm{~h}$ of reflux. The mixture was cooled to 0 ${ }^{\circ} \mathrm{C}$ and then water $(1 \mathrm{~mL}), \mathrm{NaOH} 15 \%(1 \mathrm{~mL})$ and water $(4 \mathrm{~mL})$ were added. The mixture was stirred over night under argon atmosphere. The white precipitate was filtered off and extracted with warm THF, and the volatiles were removed under vacuum to give a green oil that was acidified with $\mathrm{HCl} 10 \%$. The solution was extracted three times with dichloromethane. The organic phase was dried over anhydrous $\mathrm{MgSO}_{4}$ and the solvent was removed under vacuum to give a red solid in a final yield of $39 \%$.

${ }^{1} \mathrm{H}$ NMR $\left(200 \mathrm{MHz}, \mathrm{CDCl}_{3}\right) \delta 6.72$ (s, 2H), 3.66 (br s, 2H), 2.83 (s, 6H). MS (EI): calc. for $\mathrm{C}_{8} \mathrm{H}_{10} \mathrm{Cl}_{2} \mathrm{~N}_{2}$ : 204, found: 204 . 


\section{$\underline{\operatorname{Lig}^{3} \mathrm{H}_{2}}$}

A solution of 2-(bromomethyl)-4,6-bis(1,1-dimethylethyl)phenol (0.84 g, $2.8 \mathrm{mmol})$ in dry THF (5 mL) was added to a stirred solution of 4,5-dichloro- $N, N^{\prime}$-dimethyl-1,2-diaminobenzene $(0.30 \mathrm{~g}, 1.4 \mathrm{mmol})$ in dry THF $(5 \mathrm{~mL})$ under argon atmosphere. A solution of triethylamine $(0.5 \mathrm{~mL})$ in dry THF $(2 \mathrm{~mL})$ was added dropwise and a precipitate had formed. The reaction mixture was stirred for $2 \mathrm{~h}$ in the dark under argon atmosphere. The purple precipitate was removed, and the solvent of the brown filtrate was removed under vacuum forming a brown solid. The solid was redissolved in dichloromethane and washed twice with $\mathrm{NaCl}$ solution. The organic phase was dried over anhydrous $\mathrm{MgSO}_{4}$ and the solvent was removed under vacuum to give the crude product as a brown solid. The crude product was recrystallized from cold methanol to give an off-white solid that was further purified by flash chromatography, on Silica gel $60 \mathrm{H}$ with chloroform as eluent. The pure product was obtained as an offwhite solid in a final yield of $38 \%$.

M.p. $92{ }^{\circ} \mathrm{C}$ (decomposed). ${ }^{1} \mathrm{H}$ NMR (400 MHz, $\left.\mathrm{C}_{6} \mathrm{D}_{6}\right) \delta 9.27$ (s, 2H), 7.42 (d, J=2.3 Hz, 2H), 7.06 (s, 2H) $6.82(\mathrm{~d}, J=2.3 \mathrm{~Hz}, 2 \mathrm{H}), 3.48(\mathrm{~s}, 4 \mathrm{H}), 2.20(\mathrm{~s}, 6 \mathrm{H}), 1.55(\mathrm{~s}, 18 \mathrm{H}), 1.32(\mathrm{~s}, 18 \mathrm{H}) .{ }^{13} \mathrm{C}$ NMR $(100.63$ $\left.\mathrm{MHz}, \mathrm{C}_{6} \mathrm{D}_{6}\right) \delta 153.7,148.0141 .5,136.7,129.5$ (10C, $C$, Arom), 128.1, 125.4, 123.8 (6C, CH, Arom), 121.9 (2C, $C$, Arom), $60.4\left(2 \mathrm{C}, C \mathrm{H}_{2}\right), 43.4\left(2 \mathrm{C}, \mathrm{NCH}_{3}\right), 35.3,34.4\left(4 \mathrm{C}, C\left(\mathrm{CH}_{3}\right)_{3}\right), 31.9,30.1(12 \mathrm{C}$ $\left.\mathrm{C}\left(\mathrm{CH}_{3}\right)_{3}\right)$. MS (FAB): calc. for $\mathrm{C}_{38} \mathrm{H}_{54} \mathrm{Cl}_{2} \mathrm{~N}_{2} \mathrm{O}_{2}$ : 641, found: 641. Anal. Calc. For $\mathrm{C}_{38} \mathrm{H}_{54} \mathrm{Cl}_{2} \mathrm{~N}_{2} \mathrm{O}_{2}$ : $\mathrm{C}_{\text {, }}$ 71.12; H, 8.48; N, 4.37. Found: C, 70.60; H, 8.56; N, 4.12.

\section{$\underline{\operatorname{Lig}^{4} \mathrm{H}_{2}}$}

A solution of 2-(bromomethyl)-4,6-dichlorophenol (0.93 g, $3.6 \mathrm{mmol})$ in dry THF (5 mL) was added to a stirred solution of 4,5-dichloro- $N, N^{\prime}$-dimethyl-1,2-diaminobenzene $(0.37 \mathrm{~g}, 1.8 \mathrm{mmol})$ in dry THF (5 $\mathrm{mL})$ under argon atmosphere. A solution of triethylamine $(0.75 \mathrm{~mL})$ in dry THF $(2 \mathrm{~mL})$ was added dropwise and a precipitate had formed. The reaction mixture was stirred for $2 \mathrm{~h}$ in the dark under argon atmosphere. The purple precipitate was removed and the solvent of the brown filtrate was removed under vacuum forming a brown solid. The crude product was purified by flash chromatography on Silica 
gel $60 \mathrm{H}$ with a mixture of petroleum ether : chloroform in increasing polarity as eluent. The pure product was obtained as an off-white solid in a final yield of $50 \%$.

M.p. $159{ }^{\circ} \mathrm{C}$ (decomposed). ${ }^{1} \mathrm{H}$ NMR (400 MHz, $\left.\mathrm{C}_{6} \mathrm{D}_{6}\right) \delta 8.06$ (br s, 2H), 7.07 (d, J=2.4 Hz, 2H), 6.88 $(\mathrm{s}, 2 \mathrm{H}), 6.76(\mathrm{~d}, J=2.4 \mathrm{~Hz}, 2 \mathrm{H}), 3.37(\mathrm{~s}, 4 \mathrm{H}), 1.97(\mathrm{~s}, 6 \mathrm{H}) .{ }^{13} \mathrm{C}$ NMR (100.63 MHz, $\left.\mathrm{C}_{6} \mathrm{D}_{6}\right) \delta 151.0$, 147.7, 129.5, (6C, $C$, Arom), 129.4, 129.3, 124.8, (6C, $C H$, Arom), 124.7, 124.5, 122.2 (6C, $C$, Arom), $58.1\left(2 \mathrm{C}, \mathrm{CH}_{2}\right), 41.0\left(2 \mathrm{C}, \mathrm{NCH}_{3}\right)$. MS (FAB): calc. for $\mathrm{C}_{22} \mathrm{H}_{18} \mathrm{Cl}_{6} \mathrm{~N}_{2} \mathrm{O}_{2}$ : 555, found: 555. Anal. Calc. For $\mathrm{C}_{22} \mathrm{H}_{18} \mathrm{Cl}_{6} \mathrm{~N}_{2} \mathrm{O}_{2}$ : C, 47.60; H, 3.27; N, 5.05. Found: C, 47.17; H, 2.91; N, 4.50.

\section{$\left.\underline{\operatorname{Lig}^{1} \operatorname{Ti}\left(\mathrm{O}^{\mathrm{i}} \mathrm{Pr}\right.}\right)_{2}$}

A solution of $\operatorname{Lig}^{1} \mathrm{H}_{2}(56 \mathrm{mg}, 0.1 \mathrm{mmol})$ in ether $(2 \mathrm{~mL})$ was added dropwise to a solution of $\mathrm{Ti}\left(\mathrm{O}^{\mathrm{i}} \mathrm{Pr}\right)_{4}$ (28 mg, $0.1 \mathrm{mmol})$ in ether $(2 \mathrm{~mL})$ at room temperature. The color changed immediately from colorless to yellow. The reaction mixture was stirred for $2 \mathrm{~h}$ and the volatiles were removed under vacuum to give $\mathrm{Lig}^{1} \operatorname{Ti}\left(\mathrm{O}^{\mathrm{i}} \mathrm{Pr}\right)_{2}$ as a yellow solid in a final yield of $86 \%$.

${ }^{1} \mathrm{H}$ NMR (400 MHz, $\left.\mathrm{C}_{6} \mathrm{D}_{6}\right) \delta 7.39(\mathrm{~d}, J=2.2 \mathrm{~Hz}, 2 \mathrm{H}), 6.65-6.72(\mathrm{~m}, 4 \mathrm{H}) 6.53(\mathrm{~d}, J=2.2 \mathrm{~Hz}, 2 \mathrm{H}), 5.15$ (hept., $J=6.0 \mathrm{~Hz}, 2 \mathrm{H}), 4.45$ (d, J=12.3 Hz, 2H), 3.17 (d, J=12.3 Hz, 2H), 2.66 (s, 6H), $1.76(\mathrm{~s}, 18 \mathrm{H}), 1.54$ $(\mathrm{d}, J=6.0 \mathrm{~Hz}, 6 \mathrm{H}), 1.28(\mathrm{~d}, J=6.0 \mathrm{~Hz} 6 \mathrm{H}), 1.20(\mathrm{~s}, 18 \mathrm{H}) .{ }^{13} \mathrm{C} \mathrm{NMR}\left(100.63 \mathrm{MHz}, \mathrm{C}_{6} \mathrm{D}_{6}\right) \delta 161.3,147.4$,

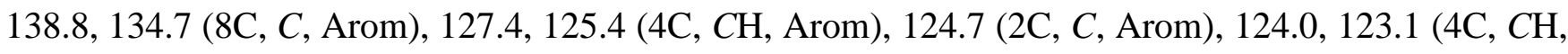
Arom), $78.1\left(2 \mathrm{C}, \mathrm{OCH}\left(\mathrm{CH}_{3}\right)_{2}\right), 65.7\left(2 \mathrm{C}, \mathrm{CH}_{2}\right), 52.4\left(2 \mathrm{C}, \mathrm{NCH}_{3}\right), 35.5,34.2\left(4 \mathrm{C}, \mathrm{C}\left(\mathrm{CH}_{3}\right)_{3}\right)$ 31.9, 31.1 $\left(12 \mathrm{C}, \mathrm{C}\left(\mathrm{CH}_{3}\right)_{3}\right), 26.9,26.2\left(4 \mathrm{C}, \mathrm{OCH}\left(\mathrm{CH}_{3}\right)_{2}\right)$. Anal. Calc. for $\mathrm{C}_{44} \mathrm{H}_{68} \mathrm{~N}_{2} \mathrm{O}_{4}$ Ti: C, 71.72; H, 9.30; N, 3.80. Found: C, 71.36; H, 9.40; N, 3.71.

\section{$\left.\underline{\operatorname{Lig}^{2} \operatorname{Ti}\left(\mathrm{O}^{\mathrm{i}} \mathrm{Pr}\right.}\right)_{2}=$}

The complex was prepared in analogy to $\mathrm{Lig}^{1} \mathrm{Ti}\left(\mathrm{O}^{\mathrm{i}} \mathrm{Pr}\right)_{2}$ from $\mathrm{Lig}^{2} \mathrm{H}_{2}(49 \mathrm{mg}, 0.1 \mathrm{mmol})$, and $\mathrm{Ti}\left(\mathrm{O}^{\mathrm{i}} \mathrm{Pr}\right)_{4}$ (28 mg, $0.1 \mathrm{mmol}) . \mathrm{Lig}^{2} \mathrm{Ti}\left(\mathrm{O}^{\mathrm{i}} \mathrm{Pr}\right)_{2}$ was obtained as a yellow solid in a final yield of $90 \%$.

${ }^{1} \mathrm{H}$ NMR $\left(400 \mathrm{MHz}, \mathrm{C}_{6} \mathrm{D}_{6}\right) \delta 7.09(\mathrm{~d}, J=2.5 \mathrm{~Hz}, 2 \mathrm{H}), 6.46-6.48(\mathrm{~m}, 2 \mathrm{H}) 6.44(\mathrm{~d}, J=2.5 \mathrm{~Hz}, 2 \mathrm{H}), 6.37-$ 6.39 (m, 2H), 5.36 (hept., $J=6.1 \mathrm{~Hz}, 2 \mathrm{H}), 4.60$ (d, J=12.8 Hz, 2H), 2.97 (d, J=12.8 Hz, 2H), 2.62 (s, 6H), 
$1.51(\mathrm{~d}, J=6.1 \mathrm{~Hz}, 6 \mathrm{H}), 1.42(\mathrm{~d}, J=6.1 \mathrm{~Hz} 6 \mathrm{H}) .{ }^{13} \mathrm{C}$ NMR $\left(100.63 \mathrm{MHz}, \mathrm{C}_{6} \mathrm{D}_{6}\right) \delta 145.8,139.1(4 \mathrm{C}, C$, Arom), 129.9, 128.5, 128.4 (6C, $C H$, Arom), 127.3 (2C, $C$, Arom), 123.2 (2C, $C H$, Arom), 122.3, 121.4 (4C, $C$, Arom), $79.9\left(2 \mathrm{C}, \mathrm{OCH}\left(\mathrm{CH}_{3}\right)_{2}\right), 64.3\left(2 \mathrm{C}, \mathrm{CH}_{2}\right), 51.3\left(2 \mathrm{C}, \mathrm{NCH}_{3}\right), 26.4,26.0\left(4 \mathrm{C}, \mathrm{OCH}\left(\mathrm{CH}_{3}\right)_{2}\right)$. Anal. Calc. for $\mathrm{C}_{28} \mathrm{H}_{32} \mathrm{Cl}_{4} \mathrm{~N}_{2} \mathrm{O}_{4}$ Ti: C, 51.72; H, 4.96; N, 4.31. Found: C, 52.01; H, 4.82; N, 4.06.

\section{$\left.\underline{\operatorname{Lig}^{3} \operatorname{Ti}\left(\mathrm{O}^{\mathrm{i}} \mathrm{Pr}\right.}\right)_{2}$.}

The complex was prepared in analogy to $\mathrm{Lig}^{1} \mathrm{Ti}\left(\mathrm{O}^{\mathrm{i}} \mathrm{Pr}\right)_{2}$ from $\mathrm{Lig}^{3} \mathrm{H}_{2}(41 \mathrm{mg}, 0.06 \mathrm{mmol})$, and $\mathrm{Ti}\left(\mathrm{O}^{\mathrm{i}} \mathrm{Pr}\right)_{4}$ (18 mg, $0.06 \mathrm{mmol}) . \mathrm{Lig}^{3} \mathrm{Ti}\left(\mathrm{O}^{\mathrm{i}} \mathrm{Pr}\right)_{2}$ was obtained as a yellow solid in a quantitative yield.

${ }^{1} \mathrm{H}$ NMR (400 MHz, C $\left.6 \mathrm{D}_{6}\right) \delta 7.41(\mathrm{~d}, J=2.4 \mathrm{~Hz}, 2 \mathrm{H}), 6.94(\mathrm{~s}, 2 \mathrm{H}) 6.51$ (d, J=2.4 Hz, 2H), 5.04 (hept., $J=6.1 \mathrm{~Hz}, 2 \mathrm{H}), 4.30(\mathrm{~d}, J=12.4 \mathrm{~Hz}, 2 \mathrm{H}), 2.87(\mathrm{~d}, J=12.5 \mathrm{~Hz}, 2 \mathrm{H}), 2.50(\mathrm{~s}, 6 \mathrm{H}), 1.74(\mathrm{~s}, 18 \mathrm{H}), 1.47$ (d, $J=6.0 \mathrm{~Hz}, 6 \mathrm{H}), 1.20(\mathrm{~s}, 18 \mathrm{H}), 1.19(\mathrm{~d}, J=6.1 \mathrm{~Hz} 6 \mathrm{H}) .{ }^{13} \mathrm{C} \mathrm{NMR}\left(100.63 \mathrm{MHz}, \mathrm{C}_{6} \mathrm{D}_{6}\right) \delta 161.2,147.5$,

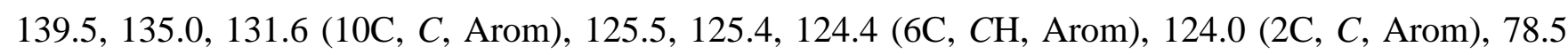
$\left(2 \mathrm{C}, \mathrm{OCH}\left(\mathrm{CH}_{3}\right)_{2}\right), 65.4\left(2 \mathrm{C}, \mathrm{CH}_{2}\right), 52.4\left(2 \mathrm{C}, \mathrm{NCH}_{3}\right), 35.6,34.2\left(4 \mathrm{C}, \mathrm{C}\left(\mathrm{CH}_{3}\right)_{3}\right)$ 31.9, 31.0 (12C, $\left.\mathrm{C}\left(\mathrm{CH}_{3}\right)_{3}\right), 26.8,26.1\left(4 \mathrm{C}, \mathrm{OCH}\left(\mathrm{CH}_{3}\right)_{2}\right)$. Anal. Calc. for $\mathrm{C}_{44} \mathrm{H}_{66} \mathrm{Cl}_{2} \mathrm{~N}_{2} \mathrm{O}_{4} \mathrm{Ti}: \mathrm{C}, 65.59 ; \mathrm{H}, 8.26 ; \mathrm{N}, 3.48$. Found: C, 65.47; H, 8.48; N, 3.22.

\section{$\left.\underline{\operatorname{Lig}^{4} \operatorname{Ti}\left(\mathrm{O}^{\mathrm{i}} \mathrm{Pr}\right.}\right)_{2}=$}

The complex was prepared in analogy to $\mathrm{Lig}^{1} \mathrm{Ti}\left(\mathrm{O}^{\mathrm{i}} \mathrm{Pr}\right)_{2}$ from $\mathrm{Lig}^{4} \mathrm{H}_{2}(44 \mathrm{mg}, 0.08 \mathrm{mmol})$, and $\mathrm{Ti}\left(\mathrm{O}^{\mathrm{i}} \mathrm{Pr}\right)_{4}$ (23 mg, $0.08 \mathrm{mmol}) . \mathrm{Lig}^{4} \mathrm{Ti}\left(\mathrm{O}^{\mathrm{i}} \mathrm{Pr}\right)_{2}$ was obtained as a yellow solid in a final yield of $91 \%$.

${ }^{1} \mathrm{H}$ NMR (400 MHz, C $\left.6 \mathrm{D}_{6}\right) \delta 7.12(\mathrm{~d}, J=2.5 \mathrm{~Hz}, 2 \mathrm{H}), 6.75(\mathrm{~s}, 2 \mathrm{H}) 6.42$ (d, J=2.5 Hz, 2H), 5.25 (hept., $J=6.1 \mathrm{~Hz}, 2 \mathrm{H}), 4.45(\mathrm{~d}, J=13.1 \mathrm{~Hz}, 2 \mathrm{H}), 2.78(\mathrm{~d}, J=13.1 \mathrm{~Hz}, 2 \mathrm{H}), 2.48(\mathrm{~s}, 6 \mathrm{H}), 1.42(\mathrm{~d}, J=6.1 \mathrm{~Hz}, 6 \mathrm{H})$, $1.35(\mathrm{~d}, J=6.1 \mathrm{~Hz} 6 \mathrm{H}) .{ }^{13} \mathrm{C} \mathrm{NMR}\left(100.63 \mathrm{MHz}, \mathrm{C}_{6} \mathrm{D}_{6}\right) \delta 158.1,145.9,132.9$ (6C, C, Arom), 130.3, 128.3, (4C, CH, Arom), 126.5 (2C, $C$, Arom), 125.5 (2C, CH, Arom), 122.5, 121.9 (4C, $C$, Arom), 80.3 $\left(2 \mathrm{C}, \mathrm{OCH}\left(\mathrm{CH}_{3}\right)_{2}\right), 64.1\left(2 \mathrm{C}, \mathrm{CH}_{2}\right), 51.2\left(2 \mathrm{C}, \mathrm{NCH}_{3}\right), 26.2,25.9\left(4 \mathrm{C}, \mathrm{OCH}\left(\mathrm{CH}_{3}\right)_{2}\right)$. Anal. Calc. for $\mathrm{C}_{28} \mathrm{H}_{30} \mathrm{Cl}_{6} \mathrm{~N}_{2} \mathrm{O}_{4} \mathrm{Ti}: \mathrm{C}, 46.76 ; \mathrm{H}, 4.20 ; \mathrm{N}, 3.90$. Found: C, 46.90; H, 3.96; N, 3.57. 


\section{$\underline{\operatorname{Lig}^{1} \mathrm{Zr}\left(\mathrm{O}^{t} \mathrm{Bu}\right)_{2}}$.}

A solution of $\mathrm{Lig}^{1} \mathrm{H}_{2}(52 \mathrm{mg}, 0.09 \mathrm{mmol})$ in ether $(2 \mathrm{~mL})$ was added dropwise to a solution of $\mathrm{Zr}\left(\mathrm{O}^{t} \mathrm{Bu}\right)_{4}$ (35 mg, $0.09 \mathrm{mmol})$ in ether $(2 \mathrm{~mL})$ at room temperature. The color changed immediately from colorless to yellow. The reaction mixture was stirred at $70{ }^{\circ} \mathrm{C}$ for $1 \mathrm{~h}$ and the volatiles were removed under vacuum to give $\operatorname{Lig}^{1} \mathrm{Zr}\left(\mathrm{O}^{t} \mathrm{Bu}\right)_{2}$ as a yellow solid in a quantitative yield.

${ }^{1} \mathrm{H}$ NMR $\left(400 \mathrm{MHz}, \mathrm{C}_{6} \mathrm{D}_{6}\right) \delta 7.38(\mathrm{~d}, J=2.2 \mathrm{~Hz}, 2 \mathrm{H}), 6.71-6.73(\mathrm{~m}, 2 \mathrm{H}) 6.64-6.66(\mathrm{~m}, 2 \mathrm{H}), 6.52(\mathrm{~d}$, $J=2.2 \mathrm{~Hz}, 2 \mathrm{H}), 4.71(\mathrm{~d}, J=12.2 \mathrm{~Hz}, 2 \mathrm{H}), 3.16(\mathrm{~d}, J=12.2 \mathrm{~Hz}, 2 \mathrm{H}), 2.63(\mathrm{~s}, 6 \mathrm{H}), 1.73$ (s, 18H), 1.55 (s, 18H), 1.19 (s, 18H). ${ }^{13} \mathrm{C}$ NMR (100.63 MHz, $\left.\mathrm{C}_{6} \mathrm{D}_{6}\right) \delta 160.2,146.7,138.0,135.5$ (8C, $C$, Arom), 127.5, 126.2, 124.1, 123.5 (8C, $\mathrm{CH}$, Arom), $76.3\left(2 \mathrm{C}, \mathrm{OC}\left(\mathrm{CH}_{3}\right)_{3}\right), 65.1\left(2 \mathrm{C}, \mathrm{CH}_{2}\right), 51.5\left(2 \mathrm{C}, \mathrm{NCH}_{3}\right), 35.6$, 34.1 (4C, $\left.C\left(\mathrm{CH}_{3}\right)_{3}\right)$ 33.5, 31.9, $30.8\left(18 \mathrm{C}, \mathrm{C}\left(\mathrm{CH}_{3}\right)_{3}\right)$. Anal. Calc. for $\mathrm{C}_{46} \mathrm{H}_{72} \mathrm{~N}_{2} \mathrm{O}_{4} \mathrm{Zr}$ : C, 68.35; H, 8.98; N, 3.47. Found: C, 68.62; H, 9.25; N, 3.17. Crystal data for $\mathrm{C}_{51} \mathrm{H}_{84} \mathrm{~N}_{2} \mathrm{O}_{4} \mathrm{Zr}: M=880.42$, monoclinic space group $P 2{ }_{1} / c, a=18.93630(10), b=19.1910(2), c=14.5019(2) \AA, \quad \beta=102.5869(4)^{\circ}, U=$ 5143.43(9) $\AA^{3}, Z=4, D_{\mathrm{c}}=1.137, \mu(\mathrm{Mo}-\mathrm{K} \alpha)=0.255 \mathrm{~mm}^{-1}, T=110 \mathrm{~K}, 12350$ unique reflections were measured. The final refinement converged at $R_{1}=0.0420$ and $w R_{2}=0.1054$ for $I>2 \sigma(I)$ and $R_{1}=$ 0.0559 and $w R_{2}=0.1145$ for all data.

\section{$\underline{\mathrm{Lig}^{2} \mathrm{Zr}\left(\mathrm{O}^{\mathrm{B}} \mathrm{Bu}\right)_{2}}$.}

A solution of $\mathrm{Lig}^{2} \mathrm{H}_{2}(27 \mathrm{mg}, 0.06 \mathrm{mmol})$ in ether $(2 \mathrm{~mL})$ was added dropwise to a solution of $\mathrm{Zr}\left(\mathrm{O}^{t} \mathrm{Bu}\right)_{4}$ (21 mg, $0.06 \mathrm{mmol})$ in ether $(2 \mathrm{~mL})$ at room temperature. The color changed immediately from colorless to yellow. The reaction mixture was stirred for $2 \mathrm{~h}$ and the volatiles were removed under vacuum to give $\mathrm{Lig}^{2} \mathrm{Zr}\left(\mathrm{O}^{t} \mathrm{Bu}\right)_{2}$ as a yellow solid in a final yield of $95 \%$.

${ }^{1} \mathrm{H}$ NMR (400 MHz, $\left.\mathrm{C}_{6} \mathrm{D}_{6}\right) \delta 7.07$ (d, J=2.5 Hz, 2H), 6.42-6.45 (m, 2H) 6.39 (d, J=2.5 Hz, 2H), 6.31$6.35(\mathrm{~m}, 2 \mathrm{H}), 4.64(\mathrm{~d}, J=12.7 \mathrm{~Hz}, 2 \mathrm{H}), 2.91(\mathrm{~d}, J=12.7 \mathrm{~Hz}, 2 \mathrm{H}), 2.67$ (s, 6H), 1.56 (s, 18H). ${ }^{13} \mathrm{C} \mathrm{NMR}$ $\left(100.63 \mathrm{MHz}, \mathrm{C}_{6} \mathrm{D}_{6}\right) \delta 157.1,145.2$ (4C, $C$, Arom), 130.2, 129.0, 128.5, (6C, $\mathrm{CH}$, Arom), 126.8, 123.5 (4C, $C$, Arom), 123.4 (2C, $\mathrm{CH}$, Arom), 121.0 (2C, $C$, Arom), $77.3\left(2 \mathrm{C}, \mathrm{OC}\left(\mathrm{CH}_{3}\right)_{3}\right), 63.6\left(2 \mathrm{C}, \mathrm{CH}_{2}\right)$, 
$50.2\left(2 \mathrm{C}, \mathrm{NCH}_{3}\right), 33.1\left(6 \mathrm{C}, \mathrm{C}\left(\mathrm{CH}_{3}\right)_{3}\right)$. Anal. Calc. for $\mathrm{C}_{30} \mathrm{H}_{36} \mathrm{Cl}_{4} \mathrm{~N}_{2} \mathrm{O}_{4} \mathrm{Zr}: \mathrm{C}, 49.93 ; \mathrm{H}, 5.03 ; \mathrm{N}, 3.88$. Found: C, 49.79; H, 5.10; N, 3.70.

\section{$\underline{\mathrm{Lig}^{3} \mathrm{Zr}\left(\mathrm{O}^{\mathrm{t}} \mathrm{Bu}\right)_{2}}$.}

The complex was prepared in analogy to $\mathrm{Lig}^{2} \mathrm{Zr}\left(\mathrm{O}^{\mathrm{t}} \mathrm{Bu}\right)_{2}$ from $\mathrm{Lig}^{3} \mathrm{H}_{2}$ (32 $\left.\mathrm{mg}, 0.05 \mathrm{mmol}\right)$, and $\mathrm{Zr}\left(\mathrm{O}^{\mathrm{t}} \mathrm{Bu}\right)_{4}(19 \mathrm{mg}, 0.05 \mathrm{mmol}) . \mathrm{Lig}^{3} \mathrm{Zr}\left(\mathrm{O}^{\mathrm{t}} \mathrm{Bu}\right)_{2}$ was obtained as a yellow solid in a quantitative yield.

${ }^{1} \mathrm{H}$ NMR (400 MHz, $\left.\mathrm{C}_{6} \mathrm{D}_{6}\right) \delta 7.40(\mathrm{~d}, J=2.4 \mathrm{~Hz}, 2 \mathrm{H}), 6.91(\mathrm{~s}, 2 \mathrm{H}) 6.51(\mathrm{~d}, J=2.4 \mathrm{~Hz}, 2 \mathrm{H}), 4.57(\mathrm{~d}$, $J=12.4 \mathrm{~Hz}, 2 \mathrm{H}), 2.83(\mathrm{~d}, J=12.4 \mathrm{~Hz}, 2 \mathrm{H}), 2.47(\mathrm{~s}, 6 \mathrm{H}), 1.70(\mathrm{~s}, 18 \mathrm{H}), 1.49(\mathrm{~s}, 18 \mathrm{H}), 1.20(\mathrm{~s}, 18 \mathrm{H}) .{ }^{13} \mathrm{C}$ NMR (100.63 MHz, $\left.\mathrm{C}_{6} \mathrm{D}_{6}\right) \delta 160.0,146.8,138.7,135.8,131.8$ (10C, $C$, Arom), 126.2, 125.9, 124.5 (6C, $C H$, Arom), 123.4 (2C, $C$, Arom), $76.5\left(2 \mathrm{C}, \mathrm{OC}\left(\mathrm{CH}_{3}\right)_{3}\right), 64.7\left(2 \mathrm{C}, \mathrm{CH}_{2}\right), 51.6\left(2 \mathrm{C}, \mathrm{NCH}_{3}\right), 35.6,34.1$ (4C, $\left.C\left(\mathrm{CH}_{3}\right)_{3}\right) 33.3,31.9,30.7\left(18 \mathrm{C}, \mathrm{C}\left(\mathrm{CH}_{3}\right)_{3}\right)$. Anal. Calc. for $\mathrm{C}_{46} \mathrm{H}_{70} \mathrm{Cl}_{2} \mathrm{~N}_{2} \mathrm{O}_{4} \mathrm{Zr}: \mathrm{C}, 62.98 ; \mathrm{H}, 8.04 ; \mathrm{N}$, 3.19. Found: C, 61.37; H, 8.01; N, 2.84. Crystal data for $\mathrm{C}_{46} \mathrm{H}_{70} \mathrm{Cl}_{2} \mathrm{~N}_{2} \mathrm{O}_{4} \mathrm{Zr}: M=877.16$, monoclinic space group $P 2_{1} / c, a=10.8402(2), b=29.5584(5), c=15.4830(3) \AA, \beta=100.6744(8)^{\circ}, U=$ 4875.20(15) $\AA^{3}, Z=4, D_{\mathrm{c}}=1.195, \mu(\mathrm{Mo}-\mathrm{K} \alpha)=0.375 \mathrm{~mm}^{-1}, T=110 \mathrm{~K}, 10890$ unique reflections were measured. The final refinement converged at $R_{1}=0.0565$ and $w R_{2} 0.1389$ for $I>2 \sigma(I)$ and $R_{1}=0.0922$ and $w R_{2}=0.1577$ for all data.

\section{$\underline{\operatorname{Lig}^{4} \mathrm{Zr}\left(\mathrm{O}^{t} \mathrm{Bu}\right)_{2}}$.}

The complex was prepared in analogy to $\mathrm{Lig}^{2} \mathrm{Zr}\left(\mathrm{O}^{\mathrm{t}} \mathrm{Bu}\right)_{2}$ from $\mathrm{Lig}^{4} \mathrm{H}_{2}$ (28 $\left.\mathrm{mg}, 0.05 \mathrm{mmol}\right)$, and $\mathrm{Zr}\left(\mathrm{O}^{\mathrm{t}} \mathrm{Bu}\right)_{4}(19 \mathrm{mg}, 0.05 \mathrm{mmol}) . \mathrm{Lig}^{4} \mathrm{Zr}\left(\mathrm{O}^{t} \mathrm{Bu}\right)_{2}$ was obtained as a yellow solid in quantitative yield.

${ }^{1} \mathrm{H}$ NMR (400 MHz, $\left.\mathrm{C}_{6} \mathrm{D}_{6}\right) \delta 7.09(\mathrm{~d}, J=2.5 \mathrm{~Hz}, 2 \mathrm{H}), 6.69(\mathrm{~s}, 2 \mathrm{H}) 6.37(\mathrm{~d}, J=2.5 \mathrm{~Hz}, 2 \mathrm{H}), 4.54$ (d, $J=12.9 \mathrm{~Hz}, 2 \mathrm{H}), 2.70(\mathrm{~d}, J=12.9 \mathrm{~Hz}, 2 \mathrm{H}), 2.54(\mathrm{~s}, 6 \mathrm{H}), 1.50(\mathrm{~s}, 18 \mathrm{H}) .{ }^{13} \mathrm{C} \mathrm{NMR}\left(100.63 \mathrm{MHz}, \mathrm{C}_{6} \mathrm{D}_{6}\right) \delta$ 156.9, 145.4, 133.0 (6C, $C$, Arom), 130.6, 128.9 (4C, CH, Arom), 126.1 (2C, $C$, Arom), 125.8 (2C, $C H$, Arom), 123.6, $121.6(4 \mathrm{C}, \mathrm{CH}$, Arom $), 77.6\left(2 \mathrm{C}, \mathrm{OC}\left(\mathrm{CH}_{3}\right)_{3}\right), 63.4\left(2 \mathrm{C}, \mathrm{CH}_{2}\right), 50.4\left(2 \mathrm{C}, \mathrm{NCH}_{3}\right), 33.0$ (6C, $\left.\mathrm{C}\left(\mathrm{CH}_{3}\right)_{3}\right)$. Anal. Calc. for $\mathrm{C}_{30} \mathrm{H}_{34} \mathrm{Cl}_{6} \mathrm{~N}_{2} \mathrm{O}_{4} \mathrm{Zr}$ : C, 45.58; H, 4.33; N, 3.54. Found: C, 45.51; H, 3.90; $\mathrm{N}, 3.15$. 


\section{$\underline{\operatorname{Lig}^{1} \mathrm{ZrBn}_{2}}$.}

A solution of $\operatorname{Lig}^{1} \mathrm{H}_{2}(68 \mathrm{mg}, 0.12 \mathrm{mmol})$ in toluene $(2 \mathrm{~mL})$ was added dropwise to a solution of $\mathrm{ZrBn}_{4}$ $(54 \mathrm{mg}, 0.12 \mathrm{mmol})$ in toluene $(2 \mathrm{~mL})$ at room temperature. The reaction mixture was stirred at $75^{\circ} \mathrm{C}$ for $1.5 \mathrm{~h}$ and the volatiles were removed under vacuum. The crude product was washed with pentane to give $\operatorname{Lig}^{1} \mathrm{ZrBn}_{2}$ as a yellow solid in a final yield of $61 \%$.

${ }^{1} \mathrm{H}$ NMR $\left(400 \mathrm{MHz}, \mathrm{C}_{6} \mathrm{D}_{6}\right) \delta$ 7.35-7.38 (m, 6H), $7.13(\mathrm{t}, J=7.6 \mathrm{~Hz}, 4 \mathrm{H}), 6.84(\mathrm{t}, J=7.6 \mathrm{~Hz}, 2 \mathrm{H}), 6.62-$ $6.64(\mathrm{~m}, 2 \mathrm{H}), 6.45-6.47(\mathrm{~m}, 2 \mathrm{H}), 6.35(\mathrm{~d}, J=1.9 \mathrm{~Hz}, 2 \mathrm{H}), 4.05(\mathrm{~d}, J=12.8 \mathrm{~Hz}, 2 \mathrm{H}), 3.03(\mathrm{~d}, J=10.4 \mathrm{~Hz}$, 2H), $2.83(\mathrm{~d}, J=12.8 \mathrm{~Hz}, 2 \mathrm{H}), 2.59(\mathrm{~d}, J=10.4 \mathrm{~Hz}, 2 \mathrm{H}), 2.28(\mathrm{~s}, 6 \mathrm{H}), 1.79(\mathrm{~s}, 18 \mathrm{H}), 1.11(\mathrm{~s}, 18 \mathrm{H}) .{ }^{13} \mathrm{C}$ NMR (100.63 MHz, $\left.\mathrm{C}_{6} \mathrm{D}_{6}\right) \delta 158.8,148.8,147.0,140.6,135.6$ (10C, C, Arom), 129.0, 127.7, 127.6, 126.5 (12C, CH, Arom), 125.5 (2C, $C$, Arom), 124.3, 123.1, 121.9 (6C, CH, Arom), 69.0, 63.8 (4C, $\left.\mathrm{CH}_{2}\right), 50.2\left(2 \mathrm{C}, \mathrm{NCH}_{3}\right), 35.6,34.1\left(4 \mathrm{C} \mathrm{C}\left(\mathrm{CH}_{3}\right)_{3}\right), 31.7,30.9\left(12 \mathrm{C}, \mathrm{C}\left(\mathrm{CH}_{3}\right)_{3}\right)$.

\section{$\underline{\operatorname{Lig}^{2} \mathrm{ZrBn}_{2}}=$}

The complex was prepared in analogy to $\mathrm{Lig}^{1} \mathrm{ZrBn}_{2}$ from $\mathrm{Lig}^{2} \mathrm{H}_{2}(65 \mathrm{mg}, 0.13 \mathrm{mmol})$, and $\mathrm{ZrBn}_{4}(61$ $\mathrm{mg}, 0.13 \mathrm{mmol}$ ). $\mathrm{Lig}^{2} \mathrm{ZrBn}_{2}$ was obtained as a yellow solid in a final yield of $95 \%$.

${ }^{1} \mathrm{H}$ NMR $\left(400 \mathrm{MHz}, \mathrm{C}_{6} \mathrm{D}_{6}\right) \delta 7.27(\mathrm{~d}, J=7.6 \mathrm{~Hz}, 4 \mathrm{H}), 7.15(\mathrm{t}, J=7.4 \mathrm{~Hz}, 4 \mathrm{H}), 7.04(\mathrm{~d}, J=2.1 \mathrm{~Hz}, 2 \mathrm{H})$, $6.93(\mathrm{t}, J=7.3 \mathrm{~Hz}, 2 \mathrm{H}), 6.38-6.40(\mathrm{~m}, 2 \mathrm{H}), 6.23(\mathrm{~d}, J=2.1 \mathrm{~Hz}, 2 \mathrm{H}), 6.19-6.21(\mathrm{~m}, 2 \mathrm{H}), 3.90(\mathrm{~d}, J=13.2$ $\mathrm{Hz}, 2 \mathrm{H}), 2.58-2.64(\mathrm{~m}, 4 \mathrm{H}), 2.28(\mathrm{~s}, 6 \mathrm{H}), 2.17(\mathrm{~d}, J=9.8 \mathrm{~Hz}, 2 \mathrm{H}) .{ }^{13} \mathrm{C} \mathrm{NMR}\left(100.63 \mathrm{MHz}, \mathrm{C}_{6} \mathrm{D}_{6}\right) \delta$ 155.8, 146.1, 145.9 (6C, $C$, Arom), 130.0, 129.7, 129.4, 129.0, 128.6, 123.6 (16C, CH, Arom), 123.4, 123.2 (4C, $C$, Arom), 122.9, (2C, $C \mathrm{H}$, Arom), 65.0, $62.5\left(4 \mathrm{C}, \mathrm{CH}_{2}\right), 49.4\left(2 \mathrm{C}, \mathrm{NCH}_{3}\right)$.

\section{$\underline{\operatorname{Lig}^{3} \mathrm{ZrBn}_{2}}=$}

The complex was prepared in analogy to $\mathrm{Lig}^{1} \mathrm{ZrBn}_{2}$ from $\mathrm{Lig}^{3} \mathrm{H}_{2}$ (41 mg, $0.06 \mathrm{mmol}$ ), and $\mathrm{ZrBn}_{4}$ (29 $\mathrm{mg}, 0.06 \mathrm{mmol}$ ). $\mathrm{Lig}^{3} \mathrm{ZrBn}_{2}$ was obtained as a yellow solid in a final yield of $75 \%$.

${ }^{1} \mathrm{H}$ NMR (400 MHz, $\left.\mathrm{C}_{6} \mathrm{D}_{6}\right) \delta 7.41(\mathrm{~d}, J=2.0 \mathrm{~Hz}, 2 \mathrm{H}), 7.30(\mathrm{~d}, J=7.6 \mathrm{~Hz}, 4 \mathrm{H}), 7.09(\mathrm{t}, J=7.6 \mathrm{~Hz}, 4 \mathrm{H})$, $6.84(\mathrm{t}, J=7.3 \mathrm{~Hz}, 2 \mathrm{H}), 6.71(\mathrm{~s}, 2 \mathrm{H}), 6.33(\mathrm{~d}, J=2.0 \mathrm{~Hz}, 2 \mathrm{H}), 3.85(\mathrm{~d}, J=12.9 \mathrm{~Hz}, 2 \mathrm{H}), 2.92(\mathrm{~d}, J=10.3$ 
Hz, 2H), 2.44 (d, J=10.1 Hz, 2H), 2.42 (d, J=12.9 Hz, 2H), 2.10 (s, 6H), 1.77 (s, 18H), 1.12 (s, 18H).

${ }^{13} \mathrm{C}$ NMR $\left(100.63 \mathrm{MHz}, \mathrm{C}_{6} \mathrm{D}_{6}\right) \delta 158.7,148.1,147.2,141.2,135.8,132.1$ (12C, $C$, Arom), 129.1, 127.8, 126.6, 125.5 (12C, CH, Arom), 125.0 (2C, $C$, Arom), 124.7, 122.3 (4C, CH, Arom), 68.5, 63.5 (4C, $\left.C \mathrm{H}_{2}\right), 50.4\left(2 \mathrm{C}, \mathrm{NCH}_{3}\right), 35.6,34.2\left(4 \mathrm{C} \mathrm{C}\left(\mathrm{CH}_{3}\right)_{3}\right), 31.6,30.9\left(12 \mathrm{C}, \mathrm{C}\left(\mathrm{CH}_{3}\right)_{3}\right)$.

\section{$\underline{\operatorname{Lig}^{4} \mathrm{ZrBn}_{2}}=$}

The complex was prepared in analogy to $\mathrm{Lig}^{1} \mathrm{ZrBn}_{2}$ from $\mathrm{Lig}^{4} \mathrm{H}_{2}$ (40 mg, $0.07 \mathrm{mmol}$ ), and $\mathrm{ZrBn}_{4}$ (33 mg, $0.07 \mathrm{mmol}$ ). $\mathrm{Lig}^{4} \mathrm{ZrBn}_{2}$ was obtained as a yellow solid in a final yield of $97 \%$.

${ }^{1} \mathrm{H}$ NMR $\left(400 \mathrm{MHz}, \mathrm{C}_{6} \mathrm{D}_{6}\right) \delta 7.10-7.19(\mathrm{~m}, 8 \mathrm{H}), 7.06(\mathrm{~d}, J=2.0 \mathrm{~Hz}, 2 \mathrm{H}), 6.92(\mathrm{t}, J=7.1 \mathrm{~Hz}, 2 \mathrm{H}), 6.56(\mathrm{~s}$, 2H), 6.22 (d, J=1.8 Hz, 2H), $3.74(\mathrm{~d}, J=13.4 \mathrm{~Hz}, 2 \mathrm{H}), 2.46$ (d, J=9.8 Hz, 2H), 2.33 (d, J=13.5 Hz, 2H), $2.14(\mathrm{~s}, 6 \mathrm{H}), 2.03(\mathrm{~d}, J=9.8 \mathrm{~Hz}, 2 \mathrm{H}) .{ }^{13} \mathrm{C}$ NMR $\left(100.63 \mathrm{MHz}, \mathrm{C}_{6} \mathrm{D}_{6}\right) \delta 155.6,146.3,145.2,133.2(8 \mathrm{C}$,

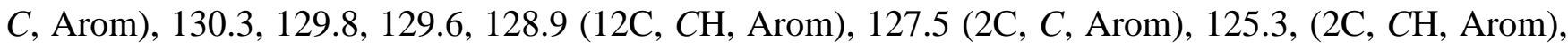
124.0 (2C, $C$, Arom), 123.9 (2C, $C H$, Arom), 123.3 (2C, $C$, Arom), 64.4, 62.2 (4C, $\left.C \mathrm{H}_{2}\right), 49.7(2 \mathrm{C}$, $\left.\mathrm{NCH}_{3}\right)$.

\section{$\underline{\text { Polymerization of neat 1-hexene employing catalysts } \mathrm{Lig}^{1} \mathrm{ZrBn}_{2}} \underline{\text { and } \mathrm{Lig}^{3} \mathrm{ZrBn}_{2}}$}

$\mathrm{B}\left(\mathrm{C}_{6} \mathrm{~F}_{5}\right)_{3}(1.2-1.3$ equiv) was dissolved in ca. $1 \mathrm{~mL}$ of 1 -hexene and added to a stirred solution of either $\operatorname{Lig}^{1} \mathrm{ZrBn}_{2}$ or $\mathrm{Lig}^{3} \mathrm{ZrBn}_{2}$ in 1-hexene at room temperature. The total volume of the monomer was ca. $5 \mathrm{~mL}$. The polymerization mixtures were left to stir at room temperature over night. The remaining 1-hexene was removed under vacuum, yielding poly(1-hexene) as a colorless sticky oil. Samples of poly(1-hexene) were analyzed by GPC and ${ }^{13} \mathrm{C}$ NMR (Table 1, Figure 1, Figure 2). The ${ }^{13} \mathrm{C}$ analysis indicated the formation of a mildly isotactic polymer $-\mathrm{mmmm}$ pentad $=65 \%$. The lack of an $\mathrm{mmrm}$ pentad and the presence of an mrrm pentad give a strong evidence for an enantiomorphic site control mechanism rather than a chain-end control mechanism (there is an overlap of the mmmr, rmmr, and mmrr pentads in poly(1-hexene) precluding their precise analysis). 
In addition, polymerization of 1-hexene employing the more active catalyst of the two $\left(\mathrm{Lig}^{3} \mathrm{ZrBn}_{2}\right)$ was followed by taking $0.50 \mathrm{~mL}$ aliquots of a $5.0 \mathrm{~mL}$ polymerization mixture every hour for six hours, removing the remaining monomer under vacuum, calculating the activity, and analyzing the resulting polymer by GPC. These results are summarized in Table 2. The broadening of PDI's with time indicated that the polymerization was not living at room temperature.

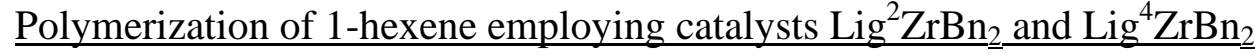

$\mathrm{B}\left(\mathrm{C}_{6} \mathrm{~F}_{5}\right)_{3}(1.2-1.3$ equiv) was dissolved in ca. $1 \mathrm{~mL}$ of 1 -hexene and added to a stirred solution of either $\operatorname{Lig}^{2} \mathrm{ZrBn}_{2}$ or $\mathrm{Lig}^{4} \mathrm{ZrBn}_{2}$ in 1-hexene at room temperature. The total volume of the monomer was ca. $5 \mathrm{~mL}$. Considerable heat had evolved and boiling of the monomer was observed within a few seconds. The following attempts were made to obtain a more well-behaved polymerization system: 1 . Reducing the catalyst quantity. 2. Increasing the volume of the monomer to $10 \mathrm{~mL}$. 3. Diluting the polymerization mixture with either 15 or $30 \mathrm{~mL}$ of an inert solvent (heptane). The most well-behaved polymerization experiments were those conducted in solution, in which no considerable temperature increase was observed and the polymerization persisted for several minutes. The remaining 1-hexene was removed under vacuum, yielding poly(1-hexene) as a colorless sticky oil, that was analyzed as

described above. ${ }^{13} \mathrm{C}$ analysis indicated the formation of a stereo-irregular polymer (Figure 3 ). The polymerization experiments in which considerable temperature rise was observed were characterized by relatively broad molecular weight distributions, whereas narrower molecular weight distributions (PDI of ca. 2) were observed for the better controlled experiments (Table 1). 
Table 1: 1-hexene polymerization data for $\mathrm{Lig}^{1-4} \mathrm{ZrBn}_{2}$

\begin{tabular}{|c|c|c|c|c|c|c|c|c|c|c|}
\hline & Pre-cat. & $\begin{array}{l}\text { Pre-cat. } \\
(\square \mathrm{mol})\end{array}$ & $\begin{array}{c}\mathrm{B}\left(\mathrm{C}_{6} \mathrm{~F}_{5}\right)_{3} \\
(\mathrm{mmol})\end{array}$ & $\begin{array}{c}1- \\
\text { hexene } \\
(\mathrm{g})\end{array}$ & $\begin{array}{c}\text { Heptane } \\
(\mathrm{mL})\end{array}$ & $\begin{array}{c}\text { Polym. } \\
\text { Time } \\
(\mathrm{min})\end{array}$ & $\begin{array}{c}\text { Polymer } \\
\text { Obtained } \\
\text { (g) }\end{array}$ & $\begin{array}{l}\text { Activity } \\
\left(\mathrm{g} \mathrm{mmol}^{-1}\right. \\
\left.\mathrm{h}^{-1}\right)\end{array}$ & $\mathrm{Mw}$ & PDI \\
\hline 1 & $\operatorname{Lig}^{1} \mathrm{ZrBn}_{2}$ & 10 & 13 & 3.30 & - & 1320 & 0.23 & 1.0 & 11,500 & 1.70 \\
\hline 2 & $\operatorname{Lig}^{2} \mathrm{ZrBn}_{2}$ & 13 & 17 & 3.07 & - & 0.5 & 2.06 & 19,000 & 47,300 & 4.3 \\
\hline 3 & $\mathrm{Lig}^{2} \mathrm{ZrBn}_{2}$ & 7 & 9 & 3.39 & 15 & 3.9 & 2.03 & 4700 & 177,000 & 2.3 \\
\hline 4 & $\mathrm{Lig}^{2} \mathrm{ZrBn}_{2}$ & 7 & 9 & 3.36 & 30 & 4.7 & 2.22 & 4300 & 177,000 & 2.4 \\
\hline 5 & $\mathrm{Lig}^{3} \mathrm{ZrBn}_{2}$ & 11 & 14 & 3.30 & - & 1260 & 1.30 & 5.6 & 37,000 & 2.2 \\
\hline 6 & $\mathrm{Lig}^{4} \mathrm{ZrBn}_{2}$ & 12 & 15 & 3.43 & - & 0.45 & 2.08 & 23,000 & 33,000 & 3.5 \\
\hline 7 & $\mathrm{Lig}^{4} \mathrm{ZrBn}_{2}$ & 6 & 8 & 6.77 & - & 0.9 & 4.51 & 50,000 & 62,000 & 2.6 \\
\hline 8 & $\mathrm{Lig}^{4} \mathrm{ZrBn}_{2}$ & 6 & 8 & 3.36 & 15 & 3.7 & 0.66 & 1,800 & 107,000 & 2.5 \\
\hline
\end{tabular}

Table 2 : 1-hexene polymerization data as a function of time for $\mathrm{Lig}^{3} \mathrm{ZrBn}_{2}$

\begin{tabular}{|c|c|c|c|c|c|}
\hline & $\begin{array}{c}\text { Polym. } \\
\text { Time } \\
(\mathrm{min})\end{array}$ & $\begin{array}{c}\text { Polymer } \\
\text { Obtained } \\
(\mathrm{mg})\end{array}$ & $\begin{array}{c}\text { Activity } \\
\left(\mathrm{g} \mathrm{mmol}^{-1}\right. \\
\left.\mathrm{h}^{-1}\right)\end{array}$ & $\mathrm{Mw}$ & PDI \\
\hline 1 & 60 & 136 & 12 & 15,000 & 1.4 \\
\hline 2 & 120 & 231 & 10.5 & 21,000 & 1.7 \\
\hline 3 & 180 & 379 & 11.5 & 25,000 & 2.0 \\
\hline 4 & 240 & 495 & 11 & 35,000 & 2.0 \\
\hline 5 & 300 & 617 & 11 & 37,000 & 2.1 \\
\hline 6 & 360 & 718 & 11 & 37,000 & 2.2 \\
\hline
\end{tabular}




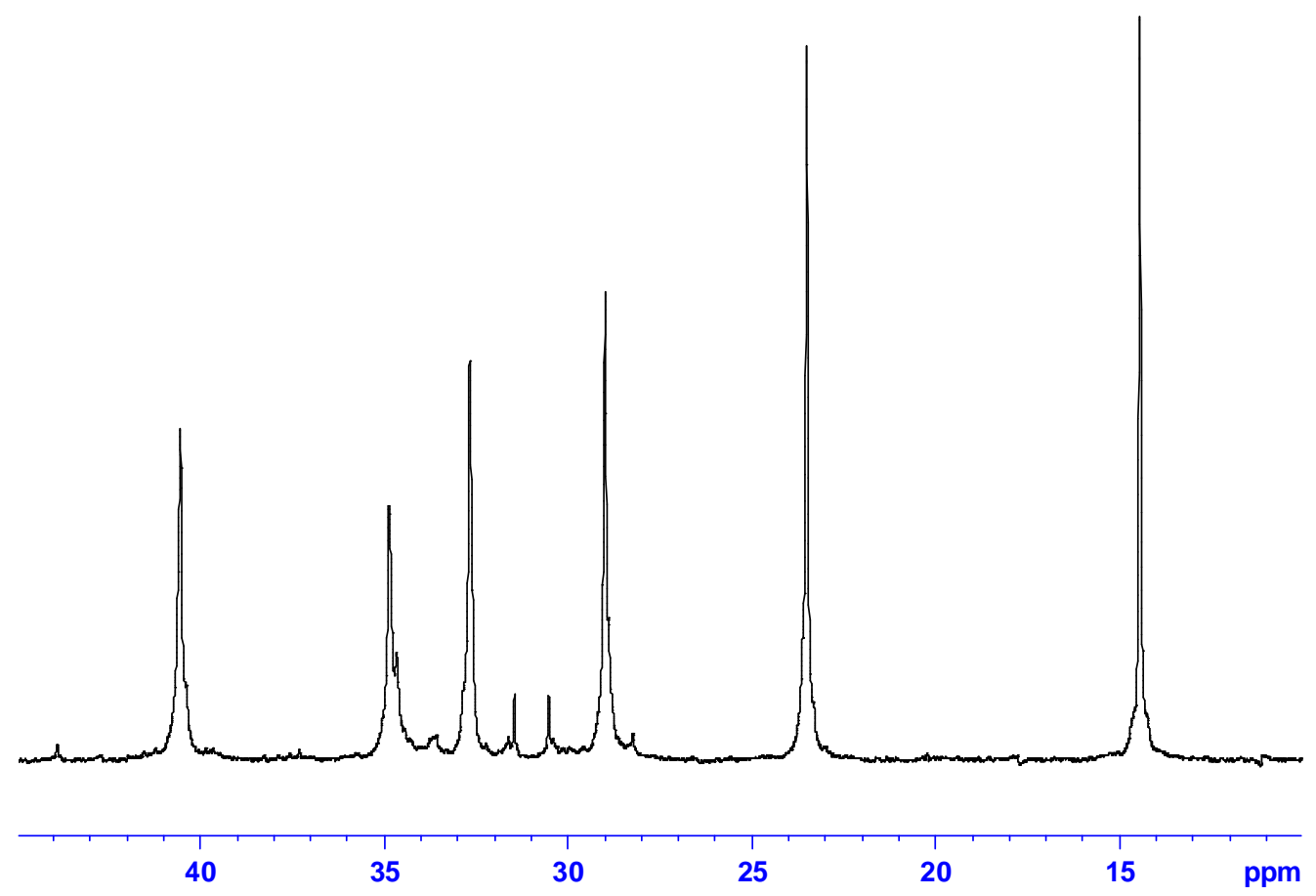

Figure 1: Isotactic poly(1-hexene) produced from $\mathrm{Lig}^{1} \mathrm{ZrBn}_{2}$.

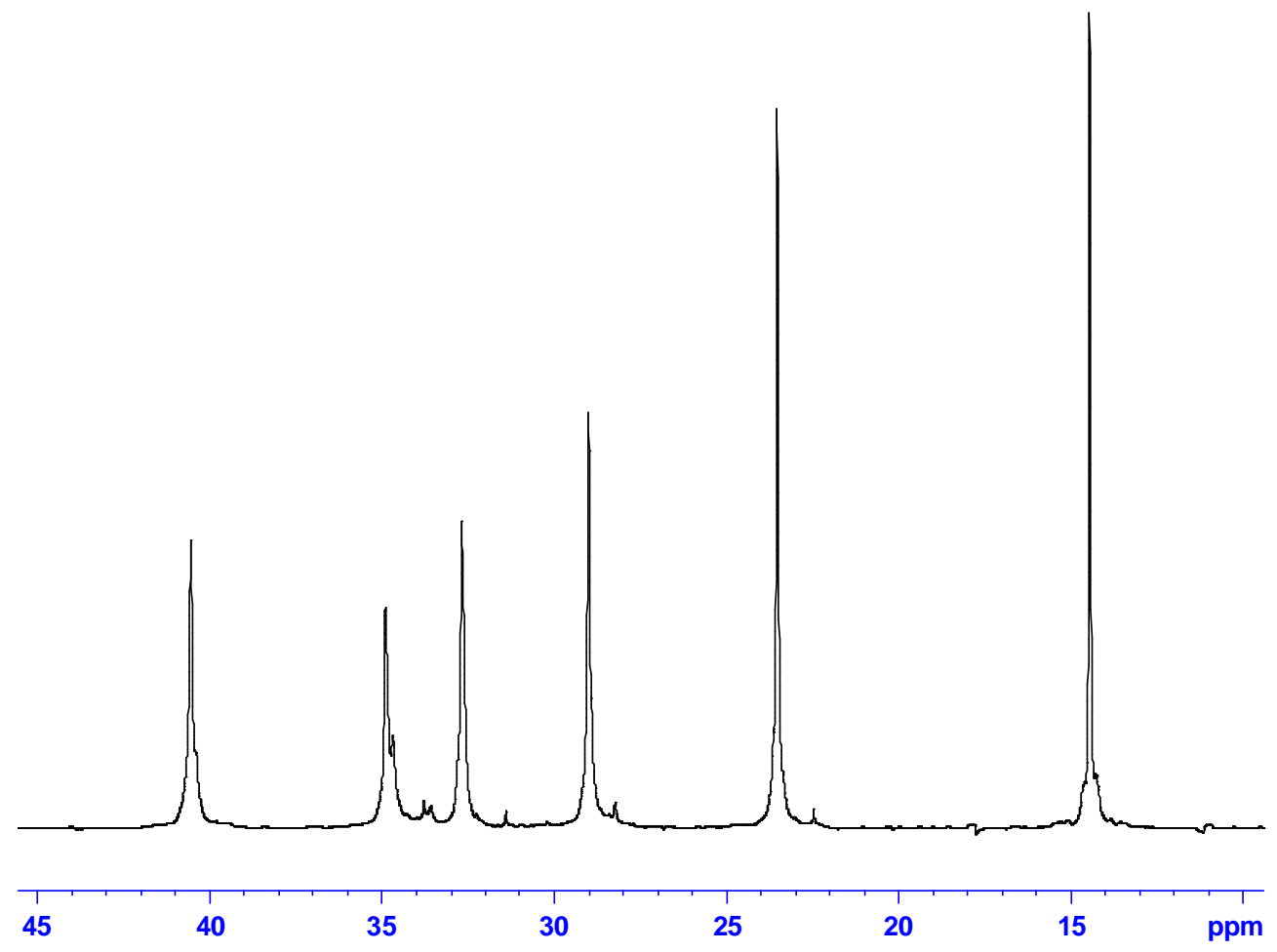

Figure 2: Isotactic poly(1-hexene) produced from $\mathrm{Lig}^{3} \mathrm{ZrBn}_{2}$. 


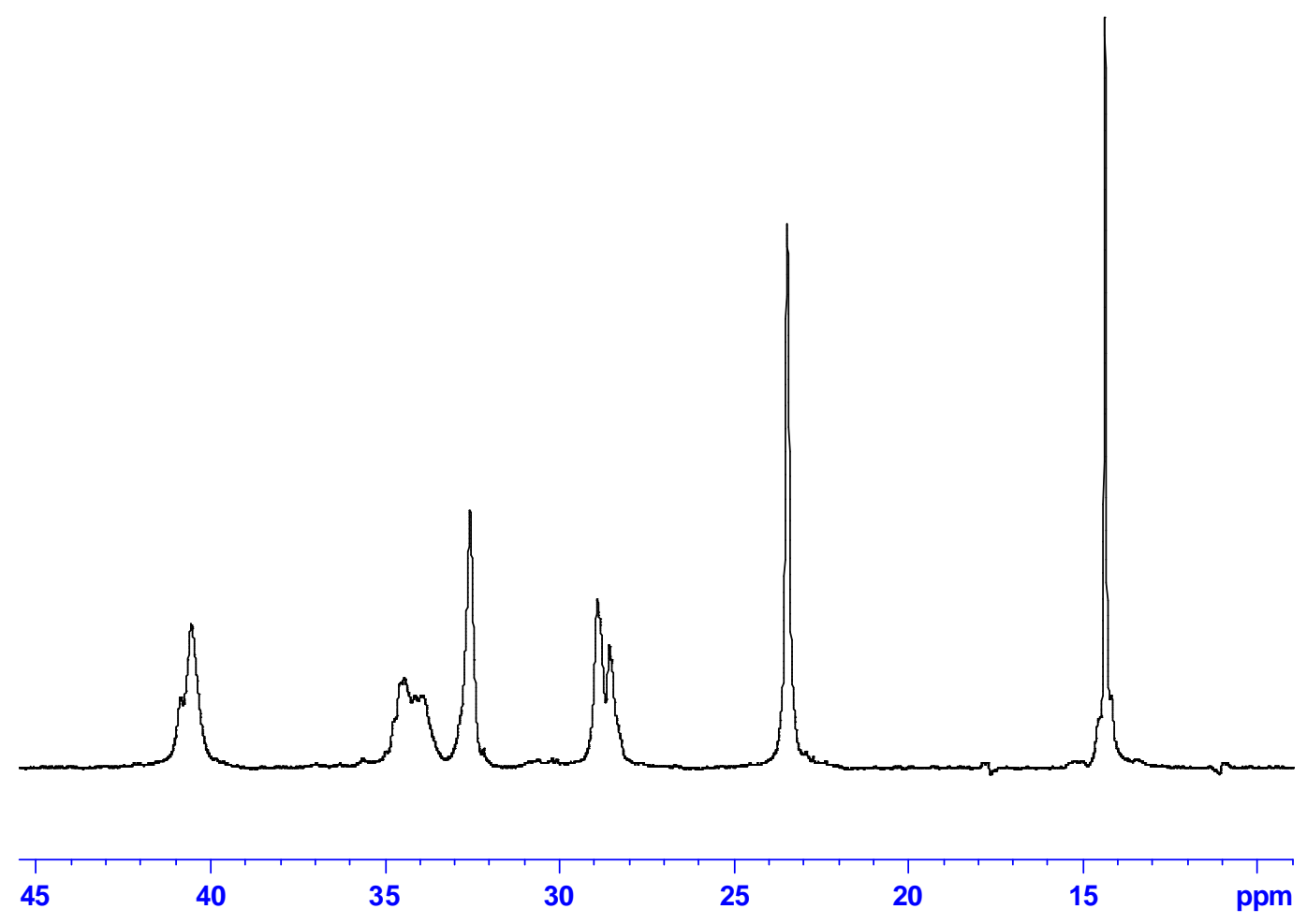

Figure 3: Atactic poly(1-hexene) produced from $\operatorname{Lig}^{4} \mathrm{ZrBn}_{2}$.

\section{References}

(1) Zucchini, U.; Alizzati, E.; Giannini, U. J. Organomet. Chem. 1971, 26, 357.

(2) Appiah, W. O.; DeGreef, A. D.; Razidlo, G. L.; Spessard, S. J.; Pink, M.; Young, V. G., Jr.; Hofmeister, G. E. Inorg. Chem. 2002, 41, 3656.

(3) Altomare, A.; Cascarano, G.; Giacovazzo, C.; Guagliardi, A.; Burla, M. C.; Polidori, G.; Camali, M. J. Appl. Cryst. 1994, 27, 435.

(4) Sheldrick, G. M. SHELXL-97 Program; University of Göttingen, Germany, 1996.

(5) Abe, N.; Ishikawa, N.; Hayashi, T.; Miura, Y. Bull. Chem. Soc. Jpn. 1990, 63, 1617.

(6) Fleming, I.; Frackenphol, J.; Ila, H. J. Chem. Soc., Perkin Trans. 1998, 1, 1229. 